\title{
Quantitative Assessment of Protective Effects of Antioxidant Agents against Drug-Induced Nephrotoxicity Using Dynamic Contrast-Enhanced Computed Tomography
}

\author{
Kenya Murase*, Akihiro Kitamura, Atsushi Tachibana, Yoshinori Kusakabe, Risa Matsuura, \\ Shohei Miyazaki
}

Department of Medical Physics and Engineering, Division of Medical Technology and Science, Faculty of Health Science, Graduate School of Medicine, Osaka University, Osaka, Japan

Email: *murase@sahs.med.osaka-u.ac.jp

How to cite this paper: Murase, K., Kitamura, A., Tachibana, A., Kusakabe, Y., Matsuura, R. and Miyazaki, S. (2016) Quantitative Assessment of Protective Effects of Antioxidant Agents against Drug-Induced Nephrotoxicity Using Dynamic Contrast-Enhanced Computed Tomography. Open Journal of Medical Imaging, 6, 53-71.

http://dx.doi.org/10.4236/ojmi.2016.63006

Received: June 12, 2016

Accepted: August 22, 2016

Published: August 25, 2016

Copyright $\odot 2016$ by authors and Scientific Research Publishing Inc. This work is licensed under the Creative Commons Attribution International License (CC BY 4.0).

http://creativecommons.org/licenses/by/4.0/ (c) (i) Open Access

\section{Abstract}

Purpose: The purpose of this study was to develop a method for quantifying the extent of renal dysfunction due to drug-induced nephrotoxicity using dynamic contrast-enhanced computed tomography (DCE-CT) and to investigate the protective effects of various antioxidant agents against cis-dichlorodiammineplatinum (cisplatin)-induced nephrotoxicity in rats using this method. Materials and Methods: The DCE-CT studies were performed in 8-week-old male Sprague-Dawley rats. The CT scanning started $4 \mathrm{~s}$ before a bolus intravenous injection of iodinated contrast agent (CA) $(150 \mathrm{mgI} / \mathrm{kg})$ from the tail vein using an automatic injector and lasted $90 \mathrm{~s}$ at 1-s intervals. The contrast clearance per unit renal volume $\left(K_{1}\right)$ was estimated from the DCE-CT data using the Patlak model. The renal volume $(V)$ was calculated by manually delineating the kidney on the CT image. The contrast clearance of the entire kidney $(K)$ was obtained by $K=K_{1} \times V$. First, to investigate the effect of CA itself, the DCE-CT studies were performed without injecting cisplatin 2, 4, and 7 days after the first DCE-CT study on day 0. Second, to investigate the effect of injected dose of cisplatin, the DCE-CT study was performed after the intraperitoneal (i.p.) injection of cisplatin $(1.8 \mathrm{mg} / \mathrm{kg})$ and was repeated every other day for one week. Finally, to investigate the protective effects of antioxidant agents [L-arginine (300 $\mathrm{mg} / \mathrm{kg}$ ), $\mathrm{N}$-acetylcysteine (500 or $1000 \mathrm{mg} / \mathrm{kg}$ ), methimazole (40 mg/kg), captopril $(60 \mathrm{mg} / \mathrm{kg})$, and taurine $(750 \mathrm{mg} / \mathrm{kg})]$, the DCE-CT studies were performed on days $0,2,4$, and 7 after the i.p. injection of cisplatin $(3.6 \mathrm{mg} / \mathrm{kg})$. For comparison, the DCE-CT data were also acquired without injecting the antioxidant agents (CDDP group). Results: When cisplatin was not injected, there were no significant changes 
in the $K$ value as compared to that on day 0 within the studied period. The $K$ valuesignificantly $(p<0.05)$ decreased with increasing dose of cisplatin. Although some differences were observed in the extent of change in the $K$ value normalized by that on day 0 , depending on the antioxidant agents and their injected dose and schedule, the normalized $K$ values on day 7 in the groups injected with the antioxidant agents were significantly higher than those in the CDDP group, suggesting that the antioxidant agents studied here had protective effects against cisplatin-induced nephrotoxicity in varying degrees. Conclusion: Our method appears useful for quantitatively evaluating the protective effects of antioxidant agents against cisplatin-induced nephrotoxicity and for investigating the optimal injected dose and schedule of the agents, because it allows repeated measurements of split renal function in a single animal.

\section{Keywords}

Dynamic Contrast-Enhanced Computed Tomography, Drug-Induced Nephrotoxicity, Cisplatin, Glomerular Filtration Rate, Creatinine Clearance, Animal Experiments

\section{Introduction}

Dynamic contrast-enhanced computed tomography (DCE-CT) acquires CT images serially after the administration of intravenous contrast agent (CA) [1]. DCE-CT has become a major imaging technique and has been widely used for evaluating the hemodynamics in normal tissue and benign and malignant tumors as well as for monitoring therapeutic effectiveness during and after therapy [1]-[3]. Dynamic contrast-enhanced magnetic resonance imaging (DCE-MRI) has also been used for studying the hemodynamics in normal and tumor tissues [4]-[6]. Although DCE-MRI provides spatial and temporal resolution comparable to DCE-CT and does not involve the use of ionizing radiation, it has some disadvantages. Whereas the change in the CT number is directly proportional to the change in the concentration of CA for DCE-CT, for DCE-MRI the relationship breaks down at high concentrations of certain CAs leading to errors of physiologic parameters [7].

Determination of glomerular filtration rate (GFR) is important in the evaluation of renal function [8]. Any substance that is filtered at the glomerulus and not reabsorbed by the renal tubules may be used as a marker of GFR. The conventional iodinated CAs have pharmacokinetics comparable to those of inulin, generally regarded as the benchmark extracellular fluid marker in physiology. Thus, values for the plasma clearance of iodinated CA are at least closely related to GFR [8].

Since DCE-CT offers the capability to measure noninvasively changes in concentration of CA in almost any regions of interest (ROIs), the potential of DCE-CT for functional renal imaging is considerable. In clinical practice, the measurement of creatinine clearance remains the most widely used method for obtaining an index of GFR. Measurement of creatinine clearance as well as any other methods with exogenous markers, 
requires a carefully timed urine collection (usually $24 \mathrm{~h}$ ) and blood sampling [8]. Radionuclide methods provide an accurate GFR measurement but their use is limited by the inherent restrictions associated with the clinical use of radioisotopes [9]. Measurements of contrast clearance using DCE-CT do not require urine collection and blood sampling, and easy to perform even on outpatients. This method will also be useful for evaluation of split renal function, and spatial resolution of CT is superior to that of radionuclide methods [8].

Cis-dichlorodiammineplatinum (cisplatin) is an important antineoplastic drug and particularly effective for the treatment of testicular and ovarian cancers [10] [11]. However, the dose-limiting nephrotoxicity of the drug is still a major clinical problem [12] [13].

Although the mechanism by which cisplatin induces renal injury is not well understood, a large body of evidence supports the concept that oxidative stress may play an important role in the pathophysiology of cisplatin-induced nephrotoxicity and that free radical generation and lipid peroxidation play a role in this toxicity [14] [15]. Accordingly, multiple trials for the reduction of nephrotoxicity have been reported using various antioxidant agents [16]-[22]. To accomplish the reduction of nephrotoxicity using antioxidant agents, it is important to quantitatively monitor the extent of renal dysfunction due to drug-induced nephrotoxicity and the effectiveness of antioxidant agents against this toxicity.

The purpose of this study was to develop a method for quantifying the extent of renal dysfunction due to drug-induced nephrotoxicity using DCE-CT, and to investigate the protective effects of various antioxidant agents against cisplatin-induced nephrotoxicity in rats using this method.

\section{Materials and Methods}

\subsection{Kinetic Model of Contrast Agent in the Kidney}

The kinetic model used for analysis consists of two compartments, i.e., the blood and the kidney. When using this model, the differential equation describing the kinetic behavior of CA in the kidney is given by [23]

$$
\frac{\mathrm{d}\left[C_{k i d}(t)-f \cdot C_{b}(t)\right]}{\mathrm{d} t}=K_{1} \cdot C_{b}(t)-k_{2}\left[C_{k i d}(t)-f \cdot C_{b}(t)\right]
$$

where $C_{\text {kid }}(t)$ and $C_{b}(t)$ are the concentrations of the CA at time $t$ in the kidney and the blood, respectively. $K_{1}$ and $k_{2}$ represent the rate constants for the transfer of CA from the blood to the kidney via glomerular filtration and outflow of the CA from the kidney, respectively. $f$ denotes the blood volume fraction.

Solving Equation (1) for $C_{k i d}(t)$ with the assumption that the initial conditions are zero and $k_{2}$ is negligible during the sampling period yields.

$$
C_{k i d}(t)=K_{1} \cdot \int_{0}^{t} C_{b}(u) \mathrm{d} u+f \cdot C_{b}(t)
$$

where $u$ is a variable of integration. Dividing both sides of Equation (2) by $C_{b}(t)$ yields. 


$$
\frac{C_{k i d}(t)}{C_{b}(t)}=K_{1} \cdot \frac{\int_{0}^{t} C_{b}(u) \mathrm{d} u}{C_{b}(t)}+f .
$$

$K_{l}$ and $f$ can be estimated from Equation (3) using the Patlak graphical method [24]. The clearance of CA in the entire kidney $(K)$ is given by

$$
K=K_{1} \cdot V
$$

where $V$ represents the renal volume. It should be noted that $K$ is the GFR in terms of the whole blood.

In this study, the time-attenuation curve (TAC) in the aorta was used as an arterial input function (AIF) instead of that in the renal artery, because it was difficult to directly obtain the TAC in the renal artery. The transit time of the CA from the aorta to the renal artery was corrected as follows. First, we manually drew the ROIs on the aorta and entire kidney as illustrated in Figure 1, and then generated the TACs in the aorta and entire kidney ROIs. Second, the transit time between the aorta and the kidney was determined by visually finding the rise points of these TACs. Finally, the transit time was corrected by shifting the TAC in the aorta to that in the entire kidney. The dispersion in AIF between the aorta and the kidney ROIs was not corrected in this study.

The partial volume effect (PVE) on $C_{b}(t)$ was corrected according to Cenic et al. [25] Briefly, a calibration curve was generated by phantom experiments containing background and contrast tubes with various diameters [23]. From the calibration curve, the recovery coefficient $(\mathrm{RC})$ value was determined, knowing the standard deviation (SD) of the gaussian fit to the background-subtracted image profile of the aorta from which the AIF was obtained [23]. The AIF corrected for the PVE was obtained by multiplying the AIF by the RC value obtained above. Figure 2(a) and Figure 2(b) show the typical example of $C_{b}(t)$ and $C_{k i d}(t)$ and Patlak plot, respectively.

Calculations were performed using MATLAB (The MathWorks, Inc., Natick, MA, USA) on a Pentium $4(2.8 \mathrm{GHz})$ computer with 2-GB RAM.

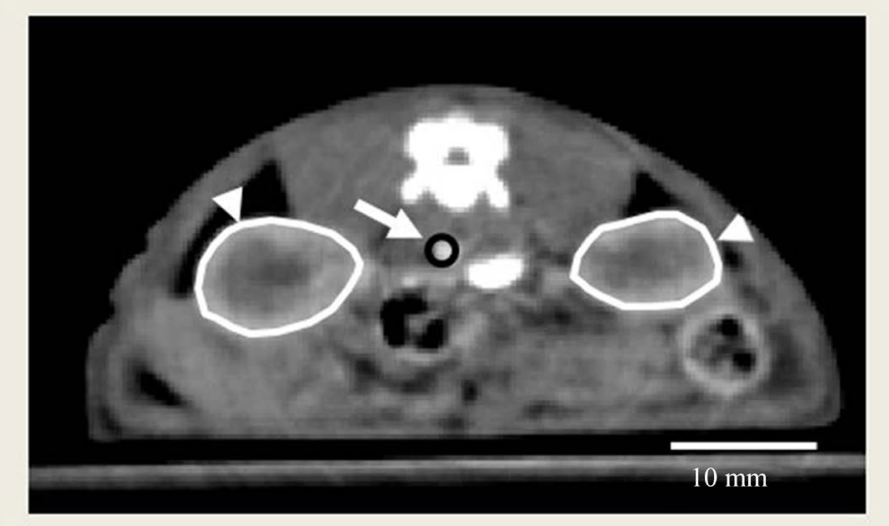

Figure 1. Example of the regions of interest (ROIs) drawn on the aorta (arrow) and entire kidneys (arrowheads) to obtain the time-attenuation curves (TACs) from dynamic contrast-enhanced computed tomography (DCE-CT) data. The TAC in the aorta ROI was used as an arterial input function. The CT image shown here was acquired $20 \mathrm{~s}$ after the start of scanning (scale bar $=10 \mathrm{~mm}$ ). 




(a)

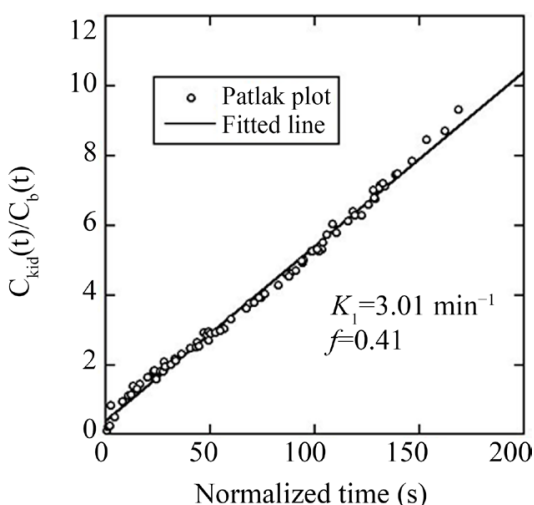

(b)

Figure 2. (a) Typical example of the TACs in the aorta $\left[C_{b}(t)\right]$ and entire kidney $\left[C_{k i d}(t)\right]$. H.U. stands for Hounsfield unit. (b) Patlak plot in which the normalized time given by $\int_{0}^{t} C_{b}(u) \mathrm{d} u / C_{b}(t)$ was taken as the $\mathrm{x}$ axis and $C_{k i d}(t) / C_{b}(t)$ was taken as the $\mathrm{y}$ axis. The rate constant for the transfer of contrast agent (CA) from the blood to the kidney via glomerular filtration $\left(K_{1}\right)$ and blood volume fraction $(f)$ were obtained from the slope and $y$-intercept of the fitted line (solid line) of the Patlak plot, respectively.

\subsection{Animal Experiments}

All animal experiments were approved by the animal ethics committee at Osaka University School of Medicine.

\subsubsection{Animals}

Male Sprague-Dawley rats were used in all experiments. They were purchased from Charles River Laboratories Japan, Inc. (Yokohama, Japan). The rats had free access to food and water, and were kept under standard laboratory conditions of 22 - 23 degree room temperatures, around 50\% humidity, and a 12:12 hour light/dark cycle.

\subsubsection{DCE-CT Protocol}

The DCE-CT studies were performed using a 4-row multi-slice CT (Asteion, Toshiba Medical Systems Co., Tochigi, Japan). For selection of the appropriate transverse level for DCE-CT studies, unenhanced scout scanning through the kidneys was performed with a tube voltage of $120 \mathrm{kV}$ and a tube current of $80 \mathrm{~mA}$. After selection of the transverse level, the DCE-CT study was performed with the same tube voltage and tube current used for scout scanning. The matrix size was $512 \times 512$ and the field of view (FOV) was $90 \mathrm{~mm} \times 90 \mathrm{~mm}$. Four slices with a thickness of $3 \mathrm{~mm}$ were acquired with a gantry rotation speed of $1.5 \mathrm{~s}$.

For the dynamic study, CT scanning was initiated $4 \mathrm{~s}$ before administration of CA. A bolus of $150 \mathrm{mgI} / \mathrm{kg}$ of iodinated CA [Iopamiron $300(300 \mathrm{mgI} / \mathrm{mL})$, Bayer Schering Pharma, Osaka, Japan] was intravenously administered at a rate of $0.125 \mathrm{~mL} / \mathrm{s}$ for $2 \mathrm{~s}$ via the 26-gauge tail vein catheter using an automatic injector of our own manufacture. Dynamic scanning was continued for $90 \mathrm{~s}$, and transverse images were reconstructed at 1-s intervals, resulting in 90 consecutive images per slice.

After the DCE-CT scanning, helical CT scanning covering the entire kidneys was 
performed with the same tube voltage, tube current, and FOV used for DCE-CT scanning, a slice thickness of $1 \mathrm{~mm}$, and a table speed of $5.5 \mathrm{~mm} /$ rotation, i.e., a helical pitch of 5.5 for the measurement of renal volumes.

\subsubsection{Measurement of Renal Volume}

The renal volume [ $V$ in Equation (4)] of each kidney was measured by helical CT after the DCE-CT study as described above. The kidney was continuously scanned from upper pole to lower pole, using 1-mm thickness and 1-mm interval. Renal contours were identified from each tomographic level and the surrounded areas were multiplied by slice thickness $(1 \mathrm{~mm})$ to yield global renal volume.

\subsubsection{Measurement of Creatinine Clearance}

Endogenous creatinine clearance $\left(C_{\mathrm{cr}}\right)$ was measured as follows. First, rats were housed in metabolic cages for $24 \mathrm{~h}$ and urine was collected one day before the last DCE-CT study. After the last DCE-CT study, blood samples were collected by heart puncture and used for the hematocrit measurement. Serum was separated from blood samples by centrifugation at $6200 \mathrm{rpm}$ for $10 \mathrm{~min}$ and used for the determination of creatinine. The kidneys were removed for histopathological evaluation. The $C_{\text {cr }}$ was calculated according to the standard formula $C_{c r}=U_{c r} \times V_{u} / P_{c r}$ in which $U_{c r}$ is the creatinine concentration in the $24 \mathrm{~h}$ urine portion, $V_{u}$ is the volume of the urine for one minute, and $P_{c r}$ is the plasma concentration of creatinine measured in the same time period [26] [27].

\subsection{Effect of Contrast Agent}

A total of 6 rats ( 8 weeks old) weighing $284 \pm 4 \mathrm{~g}$ (mean $\pm \mathrm{SD}$ ) were used to investigate the effect of CA itself. The DCE-CT studies were performed on days $0,2,4$, and 7. Day 0 was defined as the day of the first DCE-CT study. When performing the DCE-CT studies, the animal was anaesthetized using pentobarbital sodium (Somnopentyl, Kyoritsu Seiyaku Co., Tokyo, Japan) at a dose of $50 \mathrm{mg} / \mathrm{kg}$ and was placed on the CT table in a prone position. To reduce the physiological motion such as respiratory motion, we immobilized the animal with surgical tape.

\subsection{Effect of Injected Dose of Cisplatin}

A total of 36 rats ( 8 weeks old) weighing $283 \pm 8 \mathrm{~g}$ (mean $\pm \mathrm{SD}$ ) were used to investigate the effect of injected dose of cisplatin. The rats were divided into a control $(n=9)$ and cisplatin-treated groups $(\mathrm{n}=27)$. The rats in the cisplatin-treated group were given $1.8 \mathrm{mg} / \mathrm{kg}$ of cisplatin (Nippon Kayaku Co., Tokyo, Japan) intraperitoneally (i.p.) every other day [23], and were divided into three groups based on the number of injections; two-times treated $(n=9)$, four-times treated $(n=9)$, and six-times treated groups $(n=$ 9). On the day after the last cisplatin injection, the DCE-CT studies were performed. The rats in the control group were i.p. injected with saline $(3.8 \mathrm{~mL} / \mathrm{kg})$ and then the DCE-CT study was performed. Because the injected volume of saline ranged from 2.5 to $5 \mathrm{~mL} / \mathrm{kg}$ in [18] [20] [22], this injected volume was determined by averaging these 
values.

\subsection{Protective Effects of Antioxidant Agents}

In this study, L-arginine (L-ARG), N-acetylcysteine (NAC), methimazole, captopril, and taurine were used as antioxidant agents. $\mathrm{N}^{\mathrm{G}}$-nitro-L-arginine methyl ester (L-NAME) was used as a nitric oxide synthase inhibitor. L-ARG, L-NAME, NAC, methimazole, and taurine were purchased from Sigma-Aldrich Japan Co. (Tokyo, Japan). Captopril was purchased from Tokyo Chemical Industry Co. (Tokyo, Japan). Unless specifically stated, the DCE-CT studies were performed on days $0,2,4$, and 7 . Day 0 was defined as the day of cisplatin injection.

\subsubsection{Treatment with Cisplatin Alone}

A total of 15 rats ( 8 weeks old) weighing $290 \pm 7 \mathrm{~g}$ (mean $\pm \mathrm{SD}$ ) were used to investigate the effect of cisplatin alone. They were divided into two groups [CDDP (1) and CDDP (2)]. The rats in the CDDP (1) group were i.p. injected with a single dose of cisplatin $(3.6 \mathrm{mg} / \mathrm{kg})$ on day 0 and were injected with saline $(3.8 \mathrm{~mL} / \mathrm{kg})$ every day thereafter $(n=6)$. The rats in the CDDP (2) group were i.p. injected with a single dose of cisplatin $(3.6 \mathrm{mg} / \mathrm{kg})$ alone $(\mathrm{n}=9)$.

The $K$ values normalized by those on day 0 in the CDDP (1) group were compared with those in the groups injected with L-ARG, L-NAME, methimazole, captopril, and taurine in addition to cisplatin, whereas thenormalized $K$ values in the CDDP (2) group were compared with those in the group injected with cisplatin and NAC.

\subsubsection{Treatment with L-ARG and L-NAME}

A total of 18 rats ( 8 weeks old) weighing $295 \pm 12 \mathrm{~g}$ (mean \pm SD) were used to investigate the effects of L-ARG, L-NAME, and their combination. They were divided into three groups (ARG, NAME, and ARG-NAME), each consisting of 6 animals. The rats in the ARG group were i.p. injected with cisplatin $(3.6 \mathrm{mg} / \mathrm{kg})$ on day 0 , followed immediately by L-ARG (300 mg/kg) administration (i.p.) [16]. L-ARG (300 mg/kg) was i.p. injected every day thereafter. The rats in the NAME group were i.p. injected with cisplatin $(3.6 \mathrm{mg} / \mathrm{kg})$ on day 0 , followed immediately by L-NAME $(10 \mathrm{mg} / \mathrm{kg})$ administration (i.p.) [16]. L-NAME (10 mg/kg) was i.p. injected every day thereafter. The rats in the ARG-NAME group were i.p. injected with cisplatin $(3.6 \mathrm{mg} / \mathrm{kg})$ on day 0 , followed immediately by i.p. administration of both L-ARG $(300 \mathrm{mg} / \mathrm{kg})$ and L-NAME $(10 \mathrm{mg} / \mathrm{kg})$. Both L-ARG (300 mg/kg) and L-NAME (10 mg/kg) were i.p. injected every day thereafter.

\subsubsection{Treatment with NAC}

A total of 12 rats (8 weeks old) weighing $301 \pm 12 \mathrm{~g}$ (mean $\pm \mathrm{SD}$ ) were used to investigate the effect of NAC. They were divided into two groups [NAC (500) and NAC (1000)], each consisting of 6 animals. The rats in the NAC (500) group were intravenously (i.v.) injected with NAC at a single dose of $500 \mathrm{mg} / \mathrm{kg} 15 \mathrm{~min}$ before i.p. injection of cisplatin $(3.6 \mathrm{mg} / \mathrm{kg})$ on day 0 , whereas the rats in the NAC (1000) group were i.v. injected with NAC at a single dose of $1000 \mathrm{mg} / \mathrm{kg} 15 \mathrm{~min}$ before the i.p. injection of 
cisplatin $(3.6 \mathrm{mg} / \mathrm{kg})[17]$.

\subsubsection{Treatment with Methimazole}

A total of 10 rats ( 8 weeks old) weighing $288 \pm 6$ g (mean \pm SD) were used to investigate the effect of methimazole. They were divided into two groups [Methimazole (1) and Methimazole (2)], each consisting of 5 animals. The rats in the Methimazole (1) group were i.p. injected with methimazole $(40 \mathrm{mg} / \mathrm{kg}) 30 \mathrm{~min}$ before i.p. injection of cisplatin $(3.6 \mathrm{mg} / \mathrm{kg})$ on day 0 [19]. They were i.p. injected with methimazole (40 $\mathrm{mg} / \mathrm{kg}$ ) every day after cisplatin injection. The rats in the Methimazole (2) group were i.p. injected with methimazole $(40 \mathrm{mg} / \mathrm{kg}) 30 \mathrm{~min}$ before and $30 \mathrm{~min}, 1 \mathrm{~h}, 2 \mathrm{~h}, 4 \mathrm{~h}$, and $8 \mathrm{~h}$ after i.p. injection of cisplatin $(3.6 \mathrm{mg} / \mathrm{kg})$ on day 0 [20]. They were i.p. injected with methimazole $(40 \mathrm{mg} / \mathrm{kg}$ ) every day thereafter, as in the Methimazole (1) group.

\subsubsection{Treatment with Captopril and Taurine}

Six rats (8 weeks old) weighing $291 \pm 7 \mathrm{~g}$ (mean \pm SD) were used to investigate the effect of captopril (Captopril group). They were i.p. injected with captopril (60 mg/kg) 30 min before i.p. injection of cisplatin $(3.6 \mathrm{mg} / \mathrm{kg})$ on day 0 [21]. After cisplatin injection, they were i.p. injected with captopril $(60 \mathrm{mg} / \mathrm{kg})$ every day.

Six rats ( 8 weeks old) weighing $281 \pm 7 \mathrm{~g}$ (mean $\pm \mathrm{SD}$ ) were used to investigate the effect of taurine (Taurine group). They were i.p. injected with taurine $(750 \mathrm{mg} / \mathrm{kg}) 1 \mathrm{~h}$ before i.p. injection of cisplatin $(3.6 \mathrm{mg} / \mathrm{kg})$ on day 0 [22]. After cisplatin injection, they were i.p. injected with taurine $(750 \mathrm{mg} / \mathrm{kg})$ every day.

\subsection{Histopathological Evaluation}

After the final DCE-CT study, rats were sacrificed and kidneys were removed for histopathological evaluation. The resected kidney was sectioned in blocks, fixed in $20 \%$ formalin, then dehydrated in graded concentrations of alcohols and embedded in paraffin. The kidney block was cut into $2-\mu \mathrm{m}$ sections and stained with hematoxylin and eosin (H \& E) or periodic acid Schiff's (PAS) reagents to demonstrate polysaccharides, neutral mucopolysaccharide, and glycoproteins from epithelial tubular membranes. The histopathological observation was performed using a light microscope (ECLIPSE80i, NIKON Co. Ltd., Tokyo, Japan).

\subsection{Statistical Analysis}

Unless specifically stated, data were represented as mean \pm standard error (SE). When investigating the correlation between the $C_{\text {cr }}$ and $K$ values, regression analysis was performed and the correlation coefficient and regression equation were calculated. When investigating the effect of CA, the paired Student's $t$-test was used to test for significant differences in the $K$ values compared to those on day 0 . When investigating the effect of injected dose of cisplatin, the paired Student's $t$-test was also used to test for significant differences in the $K$ values compared to those without cisplatin injection. When investigating the effects of antioxidant agents, the $K$ values were normalized by those on day 0 and the paired Student's $t$-test was used to test for significant differences in the nor- 
malized $K$ values compared to those on day 0 . When comparing the normalized $K$ values between groups, the unpaired Student's $t$-test was used. A $p$-value less than 0.05 was considered significant.

\section{Results}

\subsection{Correlation between Creatinine Clearance and $K$ Value}

Figure 3 shows the correlation between the $C_{\mathrm{cr}}(\mathrm{x}, \mathrm{mL} / \mathrm{min})$ and $K$ values $(\mathrm{y}, \mathrm{mL} / \mathrm{min})$ in 160 kidneys selected randomly from this study. As shown in Figure 3, there was a significant $(p<0.01)$ correlation between them with a correlation coefficient of $\mathrm{r}=0.705$ and a regression equation of $\mathrm{y}=0.516 \mathrm{x}+0.388$.

\subsection{Effect of Contrast Agent}

Figure 4 shows the comparison of the $K$ values obtained from the DCE-CT studies performed without injecting cisplatin on days $0,2,4$, and 7 . Note that day 0 is the day of the first DCE-CT study. As shown in Figure 4, there were no significant differences in the $K$ values on days 2,4 , and 7 as compared to that on day 0 .

\subsection{Effect of Injected Dose of Cisplatin}

Figure 5(a) shows the $K$ values as a function of the cisplatin injection number, whereas Figure 5(b) shows the H \& E stain images in the control (upper left), two-times treated (upper right), four-times treated (lower left), and six-times treated groups (lower right). As shown in Figure 5, the $K$ value decreased (a) and the extent of renal damage increased with increasing number of cisplatin injection (b).

\subsection{Treatment with L-ARG and L-NAME}

Figure 6(a) shows the time courses of the $K$ value normalized by that on day 0 in the CDDP (1), ARG, NAME, and ARG-NAME groups. There were significant differences between the CDDP (1) and ARG groups on days 2 and 7, between the CDDP (1) and

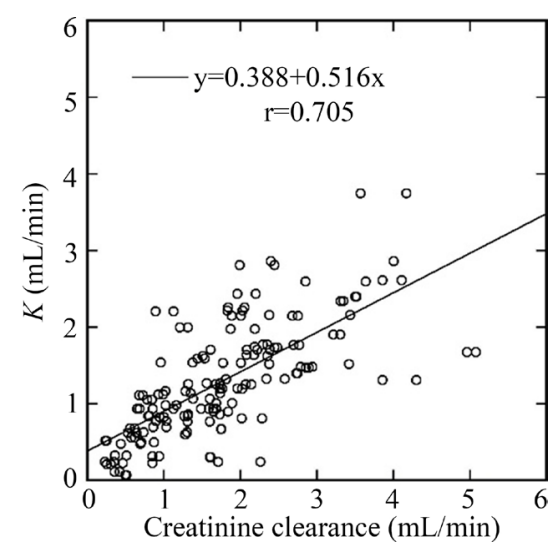

Figure 3. Relationship between creatinine clearance $\left(C_{\mathrm{cr}}\right)$ and the $K$ value in 160 kidneys selected randomly from this study. Note that the $K$ value was calculated from Equation (4) and there was a significant $(p<0.01)$ correlation between the $C_{\text {cr }}$ and $K$ values. 


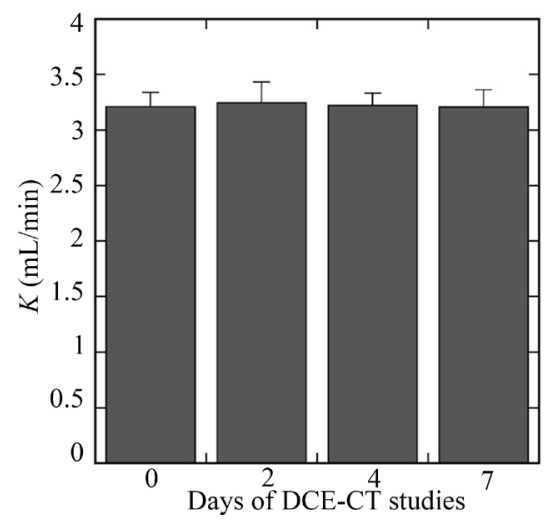

Figure 4. $K$ values obtained from the DCE-CT studies performed without injection of cisplatin on days $0,2,4$, and 7 . Note that day 0 is the day of the first DCE-CT study. Bar and error bar represent mean and standard error (SE), respectively.

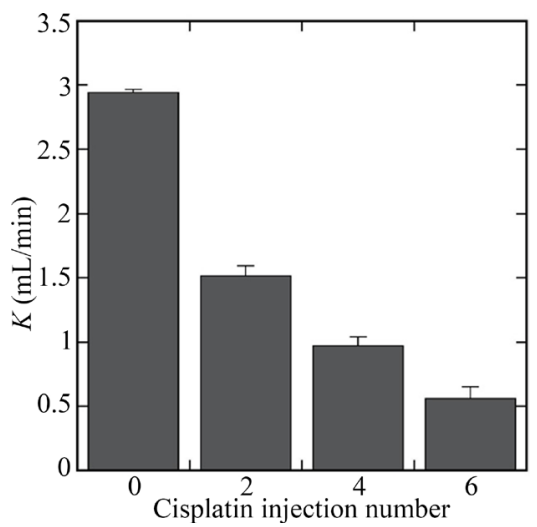

(a)

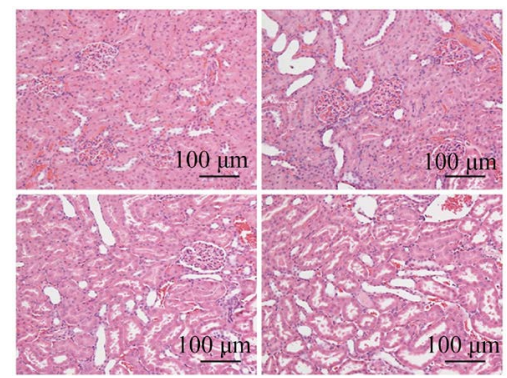

(b)

Figure 5. (a) $K$ values as a function of cisplatin injection number. Bar and error bar represent mean and SE, respectively. (b) Hematoxylin and eosin (H \& E) stain images in the control (upper left), two-times treated (upper right), four-times treated (lower left), and six-times treated groups (lower right). Magnification, $\times 200$. Scale bar $=100 \mu \mathrm{m}$.

NAME groups on day 4, and between the CDDP (1) and ARG-NAME groups on days 2 and 7. Figure 6(b) shows the PAS stain images obtained from the CDDP (1) (upper left), ARG (upper right), NAME (lower left), and ARG-NAME groups (lower right). As shown in Figure 6(b), the extent of renal damage was the most severe in the NAME group, followed by the CDDP (1), ARG-NAME, and ARG groups in descending order. 


\subsection{Treatment with NAC}

Figure 7(a) shows the time courses of the $K$ value normalized by that on day 0 in the CDDP (2), NAC (500), and NAC (1000) groups. Although there were no significant differences between the CDDP (2) and NAC (500) groups in all cases, there were significant differences between the CDDP (2) and NAC (1000) groups on days 2, 4, and 7. Figure 7(b) shows the PAS stain images obtained from the CDDP (2) (left), NAC (500) (middle), and NAC (1000) groups (right). As shown in Figure 7(b), the extent of renal damage was the most severe in the CDDP (2) group, followed by the NAC (500) and NAC (1000) groups in descending order.



(a)

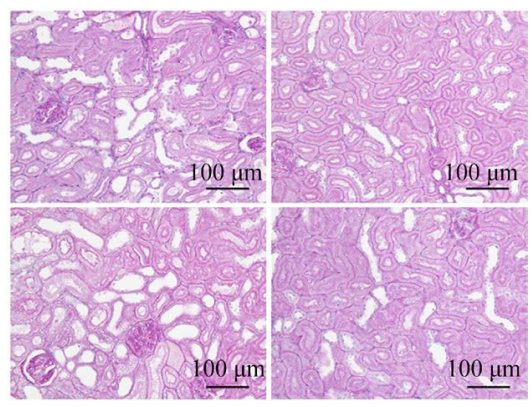

(b)

Figure 6. (a) $K$ values normalized by that on day 0 as a function of days after cisplatin injection

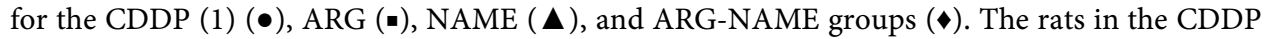
(1) group were intraperitoneally (i.p.) injected with a single dose of cisplatin $(3.6 \mathrm{mg} / \mathrm{kg})$ on day 0 and were injected with saline $(3.8 \mathrm{~mL} / \mathrm{kg})$ every day thereafter. The rats in the ARG group were i.p. injected with cisplatin $(3.6 \mathrm{mg} / \mathrm{kg})$ on day 0 , followed immediately by L-arginine (L-ARG) (300 mg/kg) administration (i.p.). L-ARG (300 mg/kg) was i.p. injected every day thereafter. The rats in the NAME group were i.p. injected with cisplatin $(3.6 \mathrm{mg} / \mathrm{kg})$ on day 0 , followed immediately by $\mathrm{N}^{\mathrm{G}}$-nitro-L-arginine methyl ester (L-NAME) (10 mg/kg) administration (i.p.). L-NAME $(10 \mathrm{mg} / \mathrm{kg}$ ) was i.p. injected every day thereafter. The rats in the ARG-NAME group were i.p. injected with cisplatin $(3.6 \mathrm{mg} / \mathrm{kg})$ on day 0 , followed immediately by i.p. administration of both L-ARG (300 mg/kg) and L-NAME (10 mg/kg). Both L-ARG (300 mg/kg) and L-NAME $\left(10 \mathrm{mg} / \mathrm{kg}\right.$ ) were i.p. injected every day thereafter. ${ }^{*}: p<0.05$ compared to the CDDP (1) group. (b) Periodic acid Schiff's (PAS) stain images in the CDDP (1) (upper left), ARG (upper right), NAME (lower left), and ARG-NAME groups (lower right). Magnification, $\times 200$. Scale bar $=100$ $\mu \mathrm{m}$. 


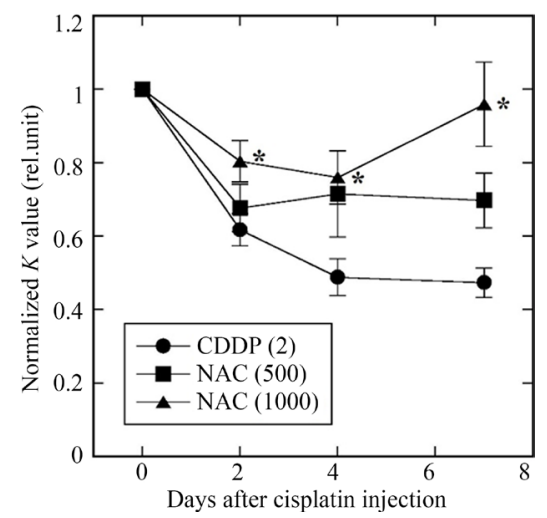

(a)

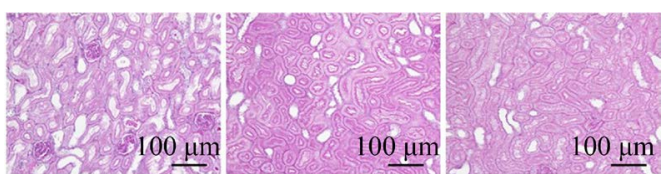

(b)

Figure 7. (a) $K$ values normalized by that on day 0 as a function of days after cisplatin injection for the CDDP (2) $(\bullet)$, NAC (500) (•), and NAC (1000) groups ( $\mathbf{\Delta})$. The rats in the CDDP (2) group were i.p. injected with a single dose of cisplatin $(3.6 \mathrm{mg} / \mathrm{kg})$ alone on day 0 . The rats in the NAC (500) group were intravenously (i.v.) injected with $\mathrm{N}$-acetylcysteine (NAC) $(500 \mathrm{mg} / \mathrm{kg})$ $15 \mathrm{~min}$ before i.p. injection of cisplatin $(3.6 \mathrm{mg} / \mathrm{kg})$ on day 0 . The rats in the NAC (1000) group were i.v. injected with NAC (1000 mg/kg) 15 min before i.p. injection of cisplatin $(3.6 \mathrm{mg} / \mathrm{kg}) \mathrm{on}$ day $0 .{ }^{*}: p<0.05$ compared to the CDDP (2) group. (b) PAS stain images in the CDDP (2) (left), NAC (500) (middle), and NAC (1000) groups (right). Magnification, $\times 200$. Scale bar $=100 \mu \mathrm{m}$.

\subsection{Treatment with Methimazole}

Figure 8(a) shows the time courses of the $K$ value normalized by that on day 0 in the CDDP (1), Methimazole (1), and Methimazole (2) groups. There were significant differences between the CDDP (1) and Methimazole (1) groups on day 7 and between the CDDP (1) and Methimazole (2) groups on days 2, 4, and 7. The normalized $K$ values in the Methimazole (2) group were significantly higher than those in the Methimazole (1) group on days 2, 4, and 7. Figure 8(b) shows the PAS stain images obtained from the CDDP (1) (left), Methimazole (1) (middle), and Methimazole (2) groups (right). The extent of renal damage was the most severe in the CDDP (1) group, followed by the Methimazole (1) and Methimazole (2) groups in descending order.

\subsection{Treatment with Captopril and Taurine}

Figure 9(a) shows the time courses of the $K$ value normalized by that on day 0 in the CDDP (1), Captopril, and Taurine groups. There were significant differences between the CDDP (1) and Captopril groups on day 7 and between the CDDP (1) and Taurine groups on days 2, 4, and 7. Figure 9(b) shows the PAS stain images obtained from the CDDP (1) (left), Captopril (middle), and Taurine groups (right). The extent of renal damage was the most severe in the CDDP (1) group. Although the extents of renal damage in the Captopril and Taurine groups were smaller than that in the CDDP (1) 




(a)

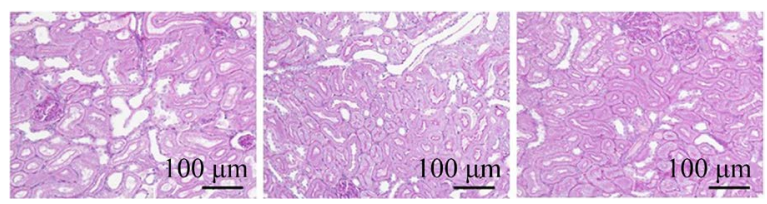

(b)

Figure 8. (a) $K$ values normalized by that on day 0 as a function of days after cisplatin injection


Methimazole (1) group were i.p. injected with methimazole $(40 \mathrm{mg} / \mathrm{kg}) 30 \mathrm{~min}$ before i.p. injection of cisplatin $(3.6 \mathrm{mg} / \mathrm{kg})$ on day 0 and every day thereafter. The rats in the Methimazole (2) group were i.p. injected with methimazole $(40 \mathrm{mg} / \mathrm{kg}) 30 \mathrm{~min}$ before and $30 \mathrm{~min}, 1 \mathrm{~h}, 2 \mathrm{~h}, 4 \mathrm{~h}$, and $8 \mathrm{~h}$ after i.p. injection of cisplatin $(3.6 \mathrm{mg} / \mathrm{kg})$ on day 0 and every day thereafter. ${ }^{*}: p<0.05$ compared to the CDDP (1) group. (b) PAS stain images in the CDDP (1) (left), Methimazole (1) (middle), and Methimazole (2) groups (right). Magnification, $\times 200$. Scale bar $=100 \mu \mathrm{m}$.

group, there was no significant difference between the Captopril and Taurine groups visually.

\section{Discussion}

In this study, we developed a method to quantitatively evaluate renal function using DCE-CT and investigated its feasibility and usefulness as a nephrotoxicity-testing tool by applying it to the evaluation of cisplatin-induced renal dysfunction in rats. We also investigated the protective effects of various antioxidant agents against cisplatininduced nephrotoxicity in rats using our method.

In the clinical setting, the measurement of $C_{\mathrm{cr}}$ remains the most widely used method for obtaining an index of GFR [26] [27]. As previously described, the measurement of the $C_{\mathrm{cr}}$, as well as any other methods with exogenous markers, requires a carefully timed urine collection (usually $24 \mathrm{~h}$ ) and blood sampling [26] [27]. Although radionuclide methods provide an accurate GFR measurement, their use is limited by the inherent restrictions associated with the clinical use of radioisotopes [9]. On the other hand, our method using DCE-CT requires neither urine collection nor blood sampling and will be easy to perform even on outpatients. Our method can also be applied to the evaluation of split renal function.

In this study, we used the Patlak model to calculate the contrast clearance per unit 


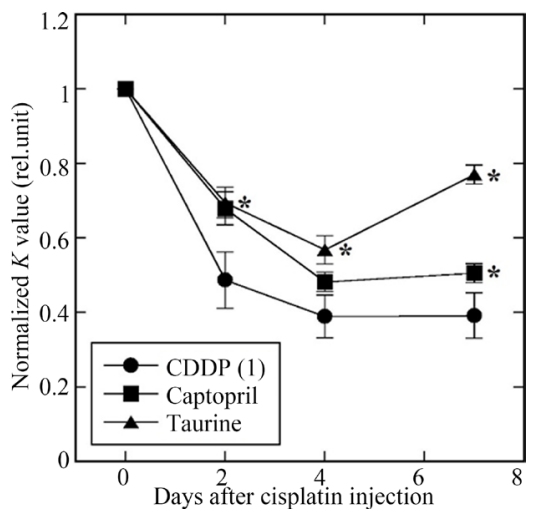

(a)

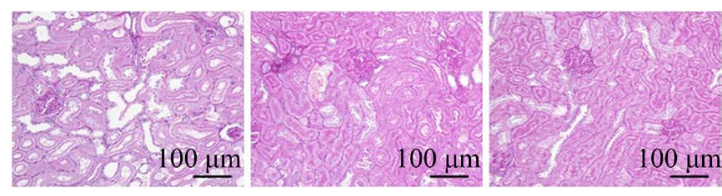

(b)

Figure 9. (a) $K$ values normalized by that on day 0 as a function of days after cisplatin injection for the CDDP (1) $(\bullet)$, Captopril (•), and Taurine groups $(\boldsymbol{\Delta})$. The rats in the Captopril group were i.p. injected with captopril $(60 \mathrm{mg} / \mathrm{kg}) 30 \mathrm{~min}$ before i.p. injection of cisplatin $(3.6 \mathrm{mg} / \mathrm{kg})$ on day 0 and every day thereafter. The rats in the Taurine group were i.p. injected with taurine $(750 \mathrm{mg} / \mathrm{kg}) 1 \mathrm{~h}$ before i.p. injection of cisplatin $(3.6 \mathrm{mg} / \mathrm{kg})$ on day 0 and every day thereafter. ${ }^{*}$ : $p<0.05$ compared to the CDDP (1) group. (b) PAS stain images in the CDDP (1) (left), Captopril (middle), and Taurine groups (right). Magnification, $\times 200$. Scale bar $=100 \mu \mathrm{m}$.

renal volume, i.e., $K_{1}$ in Equation (1) from the DCE-CT data. This model is based on the assumption that there is no outflow of the CA from the kidney during the sampling period, i.e., $k_{2}=0$ in Equation (1) [28] [29]. In addition, we used an ROI that encompassed the entire kidney including the renal cortex and medulla as illustrated in Figure 1. As shown in Figure 2(b), a good linear relationship was obtained in the Patlak plot, suggesting that the outflow of the CA from the entire kidney was negligible during the sampling period in this study. Furthermore, the $K$ value obtained from the Patlak model showed a significant correlation with $C_{\mathrm{cr}}(\mathrm{r}=0.705)$ (Figure 3$)$. These results suggest that the Patlak model is useful and appropriate for the measurement of GFR in rats. However, the slope and y-intercept of the regression line between the $C_{\mathrm{cr}}$ and $K$ values (Figure 3) were 0.516 and 0.388 , respectively. When considering the fact that $C_{\mathrm{cr}}$ derives from both the left and right kidneys, whereas the $K$ value derives from a single kidney, they were somewhat greater than the ideal slope and y-intercept, i.e., 0.5 and 0 , respectively. This may be due to the overestimation of renal volume ( $V$ ), which was used in calculating the $K$ value using Equation (4).

As previously described, we measured renal volumes by manually tracing the kidney on the CT images. To investigate the accuracy of this method, we previously compared the renal volumes measured by the manual tracing method and those measured by the formalin displacement method after sacrifice [29]. Although the renal volumes obtained by the manual tracing method significantly correlated with those measured by 
the formalin displacement method, they were overestimated by approximately $20 \%$ compared to those measured by the formalin displacement method [29]. This was probably due to the uncertainty of the renal boundary caused by partial volume effect and/or kidney movement due to physiological motion. To measure the renal volume more precisely, it would be necessary to use a more sophisticated method such as the level set method [30]. This will be one of the subjects of our future study.

To investigate the effect of CA on GFR, we performed animal experiments without injection of cisplatin (Figure 4). As shown in Figure 4, there were no significant changes in the $K$ value until day 7, indicating that the CA itself does not significantly affect the $K$ value within the studied period.

Cisplatin is a commonly used chemotherapeutic drug in the treatment of several solid tumor types [10] [11]. As previously described, however, the dose-limiting nephrotoxicity is a major clinical problem [12] [13]. The $K$ values obtained in this study decreased significantly with increasing cisplatin injection number (Figure 5(a)). In addition, the histopathological data (Figure 5(b)) showed that the renal damage due to cisplatin injection in terms of the dilatation of proximal tubules progressed in a dosedependent manner. These findings appear to support the fact that cisplatin has dosedependent renal toxicity.

Despite the fact that the underlying mechanism of cisplatin-induced nephrotoxicity is still unclear, in vitro and in vivo studies provide strong evidences that implicate oxidative stress as a mediator of cisplatin-induced nephrotoxicity [12]. Thus, we investigated the ability of various antioxidant agents (L-ARG, NAC, methimazole, captopril, and taurine) to prevent cisplatin-induced nephrotoxicity, using our method. As shown in Figure 6(a) to Figure 9(a), although some differences were observed in the extent of change in the $K$ value normalized by that on day 0 , depending on the agents and their injected dose and schedule, the normalized $K$ values on day 7 were significantly higher than those in the CDDP (1) or CDDP (2) group in all agents. Our histopathological data (Figure 5(b)) showed that the vacuolar formation and dilation of proximal tubules progressed with decreasing $K$ value. Thus, the above findings appear to indicate that the antioxidant agents studied here have protective effects against cisplatin-induced nephrotoxicity in varying degrees.

It has been reported that a nitric oxide precursor, L-ARG attenuated the oxidative stress and nephrotoxic effect of cisplatin [16]. In contrast, a nitric oxide synthase inhibitor, L-NAME was found to aggravate cisplatin-induced nephrotoxicity [16]. As shown in Figure 6(a), the normalized $K$ value in the ARG group was significantly higher than that in the CDDP (1) group 2 and 7 days after cisplatin injection, whereas the normalized $K$ value in the NAME group was significantly lower than that in the CDDP (1) group 4 days after cisplatin injection. These findings can also be confirmed by our PAS stain images (Figure 6(b)). These results appear to support the previous report [16]. Interestingly, when L-ARG and L-NAME were administered simultaneously (ARG-NAME group), the decrease in the normalized $K$ value induced by L-NAME was restored by the simultaneous administration of L-ARG, suggesting that 
nitric oxide is deeply involved in cisplatin-induced nephrotoxicity. If we combined our method with administration of drugs with various functions, it might be helpful for elucidating the underlying mechanism of drug-induced nephrotoxicity.

NAC is a precursor of glutathione and a thiol-containing antioxidant agent originally introduced as a mucolytic agent. It has been reported that NAC has beneficial effects on renal injury induced by cisplatin [17] [18]. As shown in Figure 7(a), there were significant differences in the normalized $K$ value between the CDDP (2) and NAC (1000) groups 2, 4, and 7 days after cisplatin injection. Although there was a tendency for the normalized $K$ value in the NAC (500) group to be higher than that in the CDDP (2) group, it did not reach statistical significance. These results suggest that the protective effect of NAC largely depends on the injected dose and the selection of an appropriate dose of NAC is important for enhancing the protective effect of NAC. Our method will be useful for investigating the optimal injected dose of these drugs in a preclinical stage.

Methimazole is an antithyroid drug commonly used for the treatment of hyperthyroidism. Bräunlich et al. [19] and Osman et al. [20] reported that methimazole significantly reduced the toxicity of several nephrotoxic drugs. The mechanism by which methimazole reduces the nephrotoxicity, while not entirely known, is possibly due to its antioxidant effect, resulting in the reduction of oxidative stress within the kidney [19] [20]. As shown in Figure 8(a), there were significant differences in the normalized $K$ value between the CDDP (1) and Methimazole (1) groups on day 7 and between the CDDP (1) and Methimazole (2) groups on days 2, 4, and 7. These findings support the results reported by Bräunlich et al. [19] and Osman et al. [20]. Furthermore, the normalized $K$ values in the Methimazole (2) group were always significantly higher than those in the Methimazole (1) group. These results suggest that the protective effect of methimazole against cisplatin-induced nephrotoxicity is enhanced by the multiple injection of methimazole. Thus, our method will also be useful for investigating the optimal drug administration schedule.

Angiotensin-converting enzyme (ACE) activity plays a major role in arterial hypertension and nephrotoxicity. Accordingly, ACE inhibitors such as captopril are effectively used as antihypertensive agents, and suggested to ameliorate nephrotoxicity [21]. Taurine is a non-essential amino acid produced by the body through the synthesis of two other amino acids, methionine and cysteine, and is an important component of bile acids, which are used to absorb fats and fat-soluble vitamins [31]. It also regulates heartbeat; maintains the stability of cell membranes; transports calcium in and out of cells; and regulates the activity of brain cells [31]. Taurine is also a potent antioxidant agent [22]. As shown in Figure 9(a), the normalized $K$ values in the Taurine group were significantly higher than those in the CDDP (1) group 2, 4, and 7 days after cisplatin injection. Although the normalized $K$ value in the Captopril group was significantly higher than that in the CDDP (1) group 7 days after cisplatin injection, the extent of improvement of the normalized $K$ value in this group was lower than that in the Taurine group, suggesting that taurine may be more effective against cisplatin-induced nephrotoxicity than captopril under the studied condition. These results suggest that 
our method will be helpful for selecting the optimal drug against renal injury.

\section{Conclusion}

We developed a method for quantifying renal function using DCE-CT and investigated the protective effects of various antioxidant agents (L-ARG, NAC, methimazole, captopril, and taurine) against cisplatin-induced nephrotoxicity in rats. Our results demonstrated that these antioxidant agents have protective effects against cisplatin-induced nephrotoxicity in varying degrees. Our method will be useful for quantitatively evaluating the extent of renal injury and the therapeutic effect of medication against renal injury and for investigating the optimal injected dose and schedule of medication as a tool for preclinical studies, because it allows repeated measurements of split renal function in a single animal.

\section{Declaration of Interest}

The authors report no conflicts of interest.

\section{References}

[1] Cenic, A., Nabavi, D.G., Craen, R.A., Gelb, A.W. and Lee, T.Y. (2000) A CT Method to Measure Hemodynamics in Brain Tumors: Validation and Application of Cerebral Blood Flow Maps. American Journal of Neuroradiology, 21, 462-470.

[2] Kan, Z., Phongkitkarun, S., Kobayashi, S., Tang, Y., Ellis, L.M., Lee, T.Y. and Charnsangavej, C. (2005) Functional CT for Quantifying Tumor Perfusion in Antiangiogenic Therapy in a Rat Model. Radiology, 237, 151-158. http://dx.doi.org/10.1148/radiol.2363041293

[3] Hakime, A., Peddi, H., Hines-Peralta, A.U., Wilcox, C.J., Kruskal, J., Lin, S., de Baere, T., Raptopoulos, V.D. and Goldberg, S.N. (2007) CT Perfusion for Determination of Pharmacologically Mediated Blood Flow Changes in an Animal Tumor Model. Radiology, 243, 712-719. http://dx.doi.org/10.1148/radiol.2433052048

[4] Murase, K. (2004) Efficient Method for Calculating Kinetic Parameters Using T1-Weighted Dynamic Contrast-Enhanced Magnetic Resonance Imaging. Magnetic Resonance in Medicine, 51, 858-862. http://dx.doi.org/10.1002/mrm.20022

[5] Ohno, Y., Hatabu, H., Murase, K., Higashino, T, Kawamitsu, H., Watanabe, H., Takenaka, D., Fujii, M. and Sugimura, K. (2004) Quantitative Assessment of Regional Pulmonary Perfusion in the Entire Lung Using Three-Dimensional Ultrafast Dynamic Contrast-Enhanced Magnetic Resonance Imaging: Preliminary Experience in 40 Subjects. Journal of Magnetic Resonance Imaging, 20, 353-365. http://dx.doi.org/10.1002/jmri.20137

[6] Sugawara, Y, Murase, K., Kikuchi, K., Sakayama, K., Miyazaki, T., Kajihara, M., Miki, H. and Mochizuki, T. (2006) Measurement of Tumor Blood Flow Using Dynamic Contrast-Enhanced Magnetic Resonance Imaging and Deconvolution Analysis: A Preliminary Study in Musculoskeletal Tumors. Journal of Computer Assisted Tomography, 30, 983-990. http://dx.doi.org/10.1097/01.rct.0000232475.07163.19

[7] Judd, R.M., Reeder, S.B. and May-Newman, K. (1999) Effects of Water Exchange on the Measurement of Myocardial Perfusion Using Paramagnetic Contrast Agents. Magnetic Resonance in Medicine, 41, 334-342. http://dx.doi.org/10.1002/(SICI)1522-2594(199902)41:2<334::AID-MRM18>3.0.CO;2-Y

[8] Tsushima Y. (1999) Functional CT of the Kidney. European Journal of Radiology, 30, 191- 
197. http://dx.doi.org/10.1016/S0720-048X(99)00011-X

[9] Murray, A.W., Barnfield, M.C., Waller, M.L., Telford, T. and Peters, A.M. (2013) Assessment of Glomerular Filtration Rate Measurement with Plasma Sampling: A Technical Review. Journal of Nuclear Medicine Technology, 41, 67-75.

http://dx.doi.org/10.2967/jnmt.113.121004

[10] Livingston, R.B. (1989) Cisplatin in the Treatment of Solid Tumors: Effect of Dose and Schedule. Journal of National Cancer Institute, 81, 742-745. http://dx.doi.org/10.1093/jnci/81.10.724

[11] Kuhlmann, M.K., Burkhardt, G. and Köhler, H. (1997) Insight into Potential Cellular Mechanisms of Cisplatin Nephrotoxicity and Their Clinical Application. Nephrology, Dialysis, Transplantation, 12, 2478-2480. http://dx.doi.org/10.1093/ndt/12.12.2478

[12] Ali, B.H. and Al Moundhri, M.S. (2006) Agent Ameliorating or Augmenting the Nephrotoxicity of Cisplatin and Other Platinum Compounds: A Review of Some Recent Research. Food and Chemical Toxicology, 44, 1173-1183. http://dx.doi.org/10.1016/j.fct.2006.01.013

[13] Yao, X., Panichpisal, K., Kurtzman, N. and Nugent, K. (2007) Cisplatin Nephrotoxicity: A Review. The American Journal of the Medical Sciences, 334, 115-124. http://dx.doi.org/10.1097/MAJ.0b013e31812dfe1e

[14] Hannemann, J. and Baumann K. (1988) Cisplatin-Induced Lipid Peroxidation and Decrease of Gluconeogenesis in Rat Kidney Cortex: Different Effects of Antioxidants and Radical Scavengers. Toxicology, 51, 119-132. http://dx.doi.org/10.1016/0300-483X(88)90143-6

[15] Sadzuka, Y., Shoji, T. and Takino, Y. (1992) Effect of Cisplatin on the Activities of Enzymes Which Protect against Lipid Peroxidation. Biochemical Pharmacology, 43, 1872-1875. http://dx.doi.org/10.1016/0006-2952(92)90725-X

[16] Saleh, S. and El-Demerdash, E. (2005) Protective Effects of L-Arginine against CisplatinInduced Renal Oxidative Stress and Toxicity: Role of Nitric Oxide. Basic and Clinical Pharmacology and Toxicology, 97, 91-97. http://dx.doi.org/10.1111/j.1742-7843.2005.pto_114.x

[17] Luo, J., Tsuji, T., Yasuda, H., Sun, Y., Fujigaki, Y. and Hishida, A. (2008) The Molecular Mechanisms of the Attenuation of Cisplatin-Induced Acute Renal Failure by $N$-Acetylcysteine in Rats. Nephrology, Dialysis, Transplantation, 21, 2198-2205.

http://dx.doi.org/10.1093/ndt/gfn090

[18] Dickey, D.T., Wu, Y.J., Muldoon, L.L. and Neuwelt, E.A. (2005) Protection against Cisplatin-Induced Toxicities by $N$-Acetylcysteine and Sodium Thiosulfate as Assessed at the Molecular, Cellular, and in Vivo Levels. The Journal of Pharmacology and Experimental Therapeutics, 314, 1052-1058. http://dx.doi.org/10.1124/jpet.105.087601

[19] Bräunlich, H., Appenroth, D. and Fleck, C. (1997) Protective Effects of Methimazole against Cisplatin-Induced Nephrotoxicity in Rats. Journal of Applied Toxicology, 17, 41-45. http://dx.doi.org/10.1002/(SICI)1099-1263(199701)17:1<41::AID-JAT388>3.0.CO;2-P

[20] Osman, A.M., El-Sayed, E.M., El-Demerdash, E., Al-Hyder, A., El-Didi, M., Attia, A.S. and Hamada, F.M. (2000) Prevention of Cisplatin-Induced Nephrotoxicity by Methimazole. Pharmacological Research, 41, 113-119. http://dx.doi.org/10.1006/phrs.1999.0569

[21] Mansour, M.A., El-Kashef, H.A. and Al-Shabanah, O.A. (1999) Effect of Captopril on Doxorubicin-Induced Nephrotoxicity in Normal Rats. Pharmacological Research, 39, 233-237. http://dx.doi.org/10.1006/phrs.1998.0432

[22] Erdem, A., Gündoğan, N.U., Usubütün, A., Kilinç, K., Erdem, S.R., Kara, A. and Bozkurt, A. (2000) The Protective Effect of Taurine against Gentamicin-Induced Acute Tubular Necrosis in Rats. Nephrology, Dialysis, Transplantation, 15, 1175-1182. 


\section{http://dx.doi.org/10.1093/ndt/15.8.1175}

[23] Murase, K., Tachibana, A., Kusakabe, Y., Matsuura, R. and Miyazaki, S. (2008) A Method for Quantitative Assessment of Renal Function Using Dynamic Contrast-Enhanced Computed Tomography: Evaluation of Drug-Induced Nephrotoxicity in Rats. Medical Physics, 35, 5768-5776. http://dx.doi.org/10.1118/1.3020595

[24] Patlak, C.S., Blasberg, R.G. and Fenstermacher, J.D. (1983) Graphical Evaluation of Bloodto-Brain Transfer Constants from Multiple-Time Uptake Data. Journal of Cerebral Blood Flow and Metabolism, 3, 1-7. http://dx.doi.org/10.1038/jcbfm.1983.1

[25] Cenic, A., Nabavi, D.G., Craen, R.A., Gelb, A.W. and Lee, T.Y. (1999) Dynamic CT Measurement of Cerebral Blood Flow: A Validation Study. American Journal of Neuroradiolo$g y, 20,63-73$.

[26] Cameron, J.S. (1992) Renal Function Testing. Oxford University Press, Oxford, 24-71.

[27] Levey, A.S., Madio, M.P. and Perrone, R.D. (1991) The Kidney. WB Saunders, Philadeplphia, 919-963.

[28] Hackstein, N., Puille, M.F., Bak, B.H., Scharwat, O. and Rau, W.S. (2001) Measurement of Single Kidney Contrast Media Clearance by Mutiphasic Spiral Computed Tomography: Preliminary Results. European Journal of Radiology, 39, 201-208. http://dx.doi.org/10.1016/S0720-048X(01)00323-0

[29] Murase, K., Kitamura, A., Tachibana, A., Kusakabe, Y., Matsuura, R. and Miyazaki, S. (2010) Quantitative Assessment of Early Experimental Diabetes in Rats Using Dynamic Contrast-Enhanced Computed Tomography. European Journal of Radiology, 74, 280-286. http://dx.doi.org/10.1016/j.ejrad.2009.03.006

[30] Bertalmio, M., Sapiro, G. and Randall, G. (1999) Region Tracking on Level-Sets Methods. IEEE Transactions on Medical Imaging, 18, 448-451. http://dx.doi.org/10.1109/42.774172

[31] Birdsall, T.C. (1998) Therapeutic Applications of Taurine. Alternative Medicine Review, 3, 128-136.

Submit or recommend next manuscript to SCIRP and we will provide best service for you:

Accepting pre-submission inquiries through Email, Facebook, LinkedIn, Twitter, etc. A wide selection of journals (inclusive of 9 subjects, more than 200 journals)

Providing 24-hour high-quality service

User-friendly online submission system

Fair and swift peer-review system

Efficient typesetting and proofreading procedure

Display of the result of downloads and visits, as well as the number of cited articles Maximum dissemination of your research work

Submit your manuscript at: http://papersubmission.scirp.org/ 